\title{
Angiogenic Heparin-Mimetic Peptide Nanofiber Gel Improves Regenerative Healing of Acute Wounds
}

\author{
Gozde Uzunalli, ${ }^{\dagger}$ Rashad Mammadov, ${ }^{\dagger}$ Fatih Yesildal, $^{\dagger}$ Dogan Alhan, ${ }^{\S}$ Serdar Ozturk, ${ }^{\S}$ Taner Ozgurtas, $^{\S}$ \\ Mustafa O. Guler, ${ }^{*} \dagger$ and Ayse B. Tekinay, ${ }^{\dagger} \dagger$ \\ ${ }^{\dagger}$ Institute of Materials Science and Nanotechnology, National Nanotechnology Research Center (UNAM), Bilkent University, \\ Ankara, Turkey 06800 \\ ${ }^{\ddagger}$ Department of Medical Biochemistry, Diyarbakir Military Hospital, Diyarbakir, Turkey \\ ${ }^{\S}$ Gulhane Military Medical Academy, Ankara, Turkey
}

Supporting Information

ABSTRACT: Wound repair in adult mammals typically ends with the formation of a scar, which prevents full restoration of the function of the healthy tissue, although most of the wounded skin heals. Rapid and functional recovery of major wound injuries requires therapeutic approaches that can enhance the healing process via overcoming mechanical and biochemical problems. In this study, we showed that self-assembled heparin-mimetic peptide nanofiber gel was an effective bioactive wound dressing for the rapid and functional repair of full-thickness excisional wounds in the rat model. The bioactive gel-treated wounds exhibited increased angiogenesis $(p<0.05)$, re-epithelization $(p<0.05)$, skin appendage formation, and granulation tissue organization $(p<0.05)$ compared to sucrosetreated samples. Increased blood vessel numbers in the gel-treated wounds on day 7 suggest that angiogenesis played a key role in improvement of tissue healing in bioactive gel-treated wounds. Overall, the angiogenic heparin-mimetic peptide nanofiber gel is a promising platform for enhancing the scar-free recovery of acute wounds.

KEYWORDS: heparin-mimetic peptide nanofiber, self-assembly, angiogenesis, wound healing

\section{INTRODUCTION}

Cutaneous wound healing is a morphogenic response against anything compromising tissue integrity and serves to restore the anatomic continuity and homeostasis of the affected tissue. ${ }^{1}$ As a dynamic and complex process, wound healing occurs through a series of finely orchestrated interactions between cells, extracellular elements, and signaling molecules that are present in the damaged tissue area. ${ }^{2}$ The tissue regeneration process begins immediately following any type of trauma, and is characterized by four partially overlapping steps: (i) hemostasis, (ii) inflammation, (iii) proliferation (angiogenesis, granulation, re-epithelialization), and (iv) tissue remodeling. ${ }^{3-5}$ Following the formation of a blood clot, macrophages and granulocytes infiltrate the wound area and facilitate the production of granulation tissue, which contains an extensive network of capillaries and supports the subsequent migration, proliferation, and differentiation of fibroblasts within the wound area. This process enables rapid but partial restoration of tissue functionality, and is typically completed within a few weeks. Preliminary closure of wounds is followed by a prolonged remodeling phase, which lasts $6-12$ months $^{6}$ and involves the transformation of granulation tissue into a mature scar through regression of the capillary network. ${ }^{7-9}$ Nevertheless, even mature scars can only reach about $70 \%$ of the tensile strength of normal skin. ${ }^{6}$ In addition, the amount of hair follicles and sweat and sebaceous glands, which are also called skin appendages,

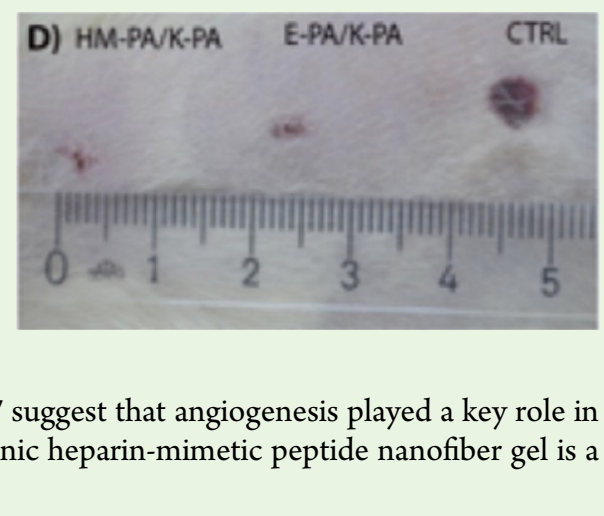


locally, and promote interaction of pro-angiogenic growth factors with their receptors. ${ }^{17-22}$ The lack of heparan sulfate groups is known to impair angiogenesis and delay the healing of wounds. In addition, binding to heparan sulfate proteoglycans protects growth factors from proteolytic degradation. ${ }^{23}$

Peptide amphiphiles are composed of bioactive peptide sequences and hydrophobic alkyl chains, and can self-assemble into nanofibrous structures through electrostatic, hydrophobic and van der Waals interactions. ${ }^{24,25}$ These molecules are frequently used in directing the lineage commitment of cells with the aid of tissue-specific bioactive signals integrated into their sequences. ${ }^{25,26}$ The nanofibrous scaffolds that are formed through self-assembly of bioactive peptide amphiphile molecules mimic the structural and functional properties of the native ECM environment, allowing great control over cellular behavior. ${ }^{27}$ These peptide nanofiber networks are biocompatible and biodegradable, which makes them highly promising materials for use as functional scaffolds in neural, ${ }^{28-30}$ bone, ${ }^{31}$ cornea, $^{32}$ enamel, ${ }^{33}$ and cartilage ${ }^{34}$ regeneration and angiogenesis. ${ }^{35}$ Peptide amphiphile nanofibers with heparin-binding groups were previously shown to enhance angiogenesis when administered in conjunction with heparin molecules. ${ }^{36,37} \mathrm{We}$ previously demonstrated heparin-mimetic peptide amphiphile (HM-PA) nanofibers, which are able to induce the formation of tubular structures by endothelial cells through their presentation of sulfonate, carboxylate and hydroxyl groups. ${ }^{38}$ Angiogenic activity was observed even in the absence of growth factors and heparin, which suggests that endogenous growth factors released from endothelial cells were sufficient for angiogenesis with the aid of heparin mimetic peptide matrix. The HM-PA was observed to induce angiogenesis both in vitro and in vivo. ${ }^{38}$ The angiogenic activity of HM-PA stems from its ability to bind heparin-binding growth factors such as vascular endothelial growth factor (VEGF) and fibroblast growth factor2 (FGF-2), thereby increasing the local concentration of these molecules. 39

Here, we tested the ability of HM-PA gels to accelerate the wound healing process in full-thickness rat skin injury model, and investigated the structural regeneration, granulation, reeptihelization, angiogenesis and wound closure rates. Because angiogenesis is a fundamental mechanism in wound healing process, the HM-PA nanofibers were able to improve the recovery of skin wounds by enhancing the formation of a blood vessel network at the wound bed. The HM-PA gel treatment provided a well-organized ECM-like environment for fullthickness wounds and increased blood vessel density and formation of skin appendages, while decreasing granulation tissue formation after 14 days of application.

\section{EXPERIMENTAL SECTION}

Materials. 9-Fluorenylmethoxycarbonyl (Fmoc) and other protected amino acids, lauric acid, 4-(2',4'-dimethoxyphenyl-Fmocaminomethyl)-phenoxyacetamido-norleucyl MBHA resin (Rink amide MBHA resin), 2-(1H-benzotriazol-1-yl)-1,1,3,3-tetramethyluronium hexafluorophosphate (HBTU), and diisopropylethylamine (DIEA) were purchased from Merck. All chemicals were used as provided.

Synthesis and Purification of Peptide Amphiphile Molecules. A Fmoc solid-phase peptide synthesis protocol was employed for the synthesis of peptide molecules. The positively charged HM-PA (lauryl-VVAGEGD(K-pbs)S-Am) and K-PA (lauryl-VVAGK-Am) were synthesized on Rink amide MBHA resin while negatively charged E-PA lauryl-VVAGE) was constructed on Wang resin. The activation of carboxylate groups was performed by 1.95 equiv. of
HBTU and 3 equiv. of DIEA for 1 molar equivalents of starting resin. Amino acid coupling time was for $2 \mathrm{~h}$. At each coupling step, samples were treated with $20 \%$ piperidine/dimethylformamide solution (DMF) for Fmoc group removal for 20 min. Unreacted amine groups were permanently blocked with $10 \%$ acetic anhydride/DMF after each coupling reaction. The resin was washed three times with DMF, dichloromethane (DCM), and DMF, respectively. A lysine residue with 4-methytrityl (Mtt) side-chain protection was used for selective deprotection of amine groups. Mtt group removal was performed by treating resins for $5 \mathrm{~min}$ with trifluoroacetic acid (TFA):triisopropylsilane (TIS): $\mathrm{H}_{2} \mathrm{O}: \mathrm{DCM}$ cocktail in the ratio of 5:2.5:2.5:90, respectively. After Mtt removal, resins were washed with DCM and DMF, respectively. Two molar equivalents of activated $p$-sulfobenzoic acid by 1.95 molar equivalents of HBTU and 3 molar equivalents of DIEA were dissolved in DMF. Coupling time was set to be $2.5 \mathrm{~h}$. The peptide amphiphile molecules were cleaved from the solid support in a TFA:TIS: $\mathrm{H}_{2} \mathrm{O}$ cocktail (95\% TFA, $2.5 \%$ water, $2.5 \%$ TIS) for $2 \mathrm{~h}$ at room temperature and excess solution was removed by rotary evaporator. Ice-cold diethyl ether was added to the remaining solution. The solution was centrifuged to obtain a white precipitate, which was dried under vacuum and reconstituted in water or culture medium prior to use. All peptide molecules synthesized in this study are watersoluble at physiological $\mathrm{pH}$. Characterization of the peptide molecules was performed by liquid chromatography and mass spectrometry (LCMS). LC-MS data were obtained using an Agilent 1200 LC system equipped with an Agilent 6530 Q-TOF with an ESI source. A Zorbax Extend-C18 $2.1 \times 50 \mathrm{~mm}$ column for basic conditions and a Zorbax SB-C8 $4.6 \mathrm{~mm} \times 100 \mathrm{~mm}$ column for acidic conditions were used for chromatographic separation. A gradient of water and acetonitrile, containing $0.1 \%$ formic acid or $0.1 \% \mathrm{NH}_{4} \mathrm{OH}$, was used. Purification of the PAs was performed by a reverse phase preparative highperformance liquid chromatography (HPLC) (Agilent 1200 series) system using a Zorbax Extend-C18 $21.2 \times 150 \mathrm{~mm}$ column. The detection wavelength was selected as $220 \mathrm{~nm}$. Concentration gradient of mobile phase was hold as $2 \%$ acetonitrile (ACN) for $2 \mathrm{~min}$ and ramp from $2 \%$ to $100 \% \mathrm{ACN}$ for $20 \mathrm{~min}$. Then, it was ramped from 100 to $2 \% \mathrm{ACN}$ and was held as $2 \% \mathrm{ACN}$ for $2 \mathrm{~min}$, sequentially.

Scanning Electron Microscopy (SEM). A FEI Quanta 200 FEG scanning electron microscope (SEM) was used to inspect the nanofiber networks of HM-PA/K-PA and E-PA/K-PA mixtures under high vacuum. One weight percent oppositely charged peptide amphiphile solutions were mixed at a ratio of $1: 1(\mathrm{v} / \mathrm{v}$ ) (final volume being $30 \mu \mathrm{L}$ ) and incubated at room temperature for $15 \mathrm{~min}$ for forming gels. Dehydration was performed by incubating the gels for 2 min in $20,40,60,80$, and $100 \%$ ethanol, sequentially. Following the ethanol exchange, samples were dried in a critical point dryer (Tousimis, Autosamdri-815B, Series C critical point dryer) in order to preserve the original morphology. Samples were coated with $6 \mathrm{~nm}$ of $\mathrm{Au} / \mathrm{Pd}$ prior to imaging.

Oscillatory Rheology. Storage and loss moduli of HM-PA/K-PA and E-PA/K-PA gels were measured at room temperature by oscillatory rheology using a $25 \mathrm{~mm}$ parallel plate (Anton Paar Physica RM301). One weight percent HM-PA/K-PA or E-PA/K-PA was loaded on the center of the lower plate at a total volume of $300 \mu \mathrm{L}$ and incubated for $15 \mathrm{~min}$ prior to measurement. A gap distance of $0.3 \mathrm{~mm}$ was used with an angular frequency range of $100-0.1 \mathrm{rad} / \mathrm{s}$ and a shear strain of $0.5 \%$.

In Vivo Wound Healing Model. In vivo experiments were carried out with 3 month old (200-250 g) Wistar rats of both sexes $(n=16)$. Animals were maintained on ad libitum access to water and nutrition in a $12 \mathrm{~h}-12 \mathrm{~h}$ light-dark cycle. All procedures concerning animals were approved by the Institutional Animal Care and Use Committee of Diskapi Yildirim Beyazit Training and Research Hospital and all experiments were performed accordingly.

Following the shaving and aseptic treatment of the dorsal area, three full-thickness excisional wounds were created on the dorsal skin of rats under general anesthesia. A $6 \mathrm{~mm}$ circular biopsy punch was used for the wounding process. First, second, and third wounds were created 2, 4 , and $6 \mathrm{~cm}$ distal to the ears, respectively. The gels were immediately applied to prevent dryness and reapplied on day 1. Final 
<smiles>CCCCCCCCCCCC(=O)NC(C(=O)NC(C(=O)NC(C)C(=O)NC(C)C(=O)NC(CCC(=O)O)C(=O)NC(C)C(=O)NC(CC(=O)O)C(=O)NC(CCCCNC(=O)c1ccc(S(=O)(=O)O)cc1)C(=O)NC(CO)C(N)=O)C(=O)NC(C(=O)O)C(C)C)C(C)C</smiles>

Lauryl-VVAGK-Am (K-PA)<smiles>CCCCCCCCCCCC(=O)NC(C(=O)N[C@H](C(=O)NC(C)C(=O)NCC(=O)NC(CCC(=O)O)C(=O)O)C(C)C)C(C)C</smiles>

Figure 1. Chemical structures of self-assembling peptide amphiphile molecules; HM-PA, E-PA, and K-PA.
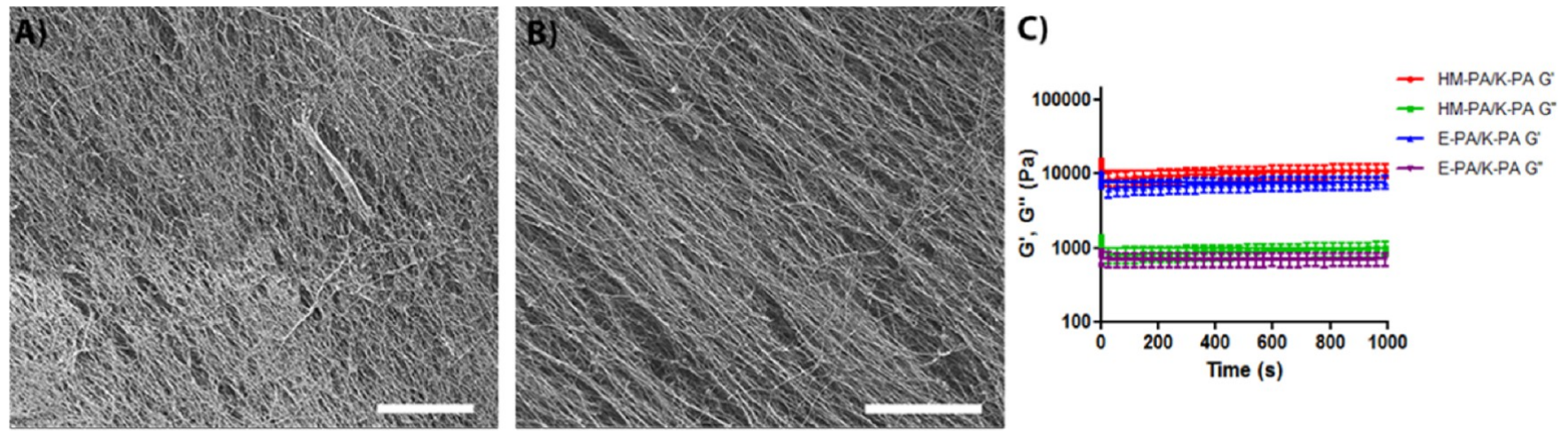

Figure 2. Characterization of the peptide amphiphile scaffolds at $\mathrm{pH}$ 7.4. Scanning electron microscopy (SEM) images of (A) HM-PA/K-PA and (B) E-PA/K-PA reveal ECM-like morphology of scaffolds. (C) Mechanical properties of the PA gels. Rheology results showed that HM-PA/K-PA and E-PA/K-PA combinations formed gels and that their mechanical properties are similar. Scale bars are $5 \mu \mathrm{m}$.

concentrations of peptide amphiphile solutions were 1 wt \% HM-PA and $1 \mathrm{wt} \% \mathrm{~K}-\mathrm{PA}$ or $1 \mathrm{wt} \% \mathrm{E}-\mathrm{PA}$ and $1 \mathrm{wt} \% \mathrm{~K}-\mathrm{PA}$, mixed at a ratio of $1: 1(\mathrm{v} / \mathrm{v})$. The E-PA/K-PA combination was used as a nonbioactive nanofiber control, and $0.25 \mathrm{M}$ sucrose solution was used as negative control. As wound location may alter the healing process through effects such as movement-mediated stretching, peptide nanofiber and control treatments were distributed evenly across distal, median and proximal wounds. Following peptide nanofiber gel application, wounds were covered with NU-DERM Alginate (Systagenix) as a wound dressing. An adhesive, elastic, nonwoven bandage (OctaCare, Octamed) was placed over the wound to prevent the spillage of gels. Rats were allowed to eat and drink ad libitum after the operation and routine controls for infection were performed throughout the study.

Wound boundaries were observed and wound areas were measured using ImageJ software (NIH, USA) on days 3, 7, 10, and 14 following the operation. Rats were euthanized at seventh $(n=8)$ and 14th $(n=$ 8 ) days following the operation and the wounds and their surrounding areas were surgically excised. Wound area closure was calculated by using the following formula

$$
\begin{aligned}
& \text { wound area ratio }(\%) \\
& =(\text { wound area on day } t / \text { wound area on day } 0) 100
\end{aligned}
$$

wound closure $(\%)=1-$ wound area ratio (\%)

Histological Analyses. Skin tissues from the sacrificed rats were collected and fixed in $10 \%$ buffered formalin. Tissues were dehydrated with $70,80,95$, and $100 \%$ ethanol series for $2 \mathrm{~h}$ each, and then they were treated with xylene and embedded in paraffin blocks. Sectioning at $5 \mu \mathrm{m}$ was performed via Leica microtome. Hematoxylin \& eosin (H\&E) and Masson's trichrome staining were performed according to standard protocols following deparaffinization and rehydration by xylene and serially decreasing concentrations of ethanol (100, 95, and $70 \%$ ethanol and water, sequentially). For immunohistochemistry experiments, the sections were labeled with anti-von Willebrand Factor (1:400; Abcam) antibody and horseradish peroxidase-conjugated goat 

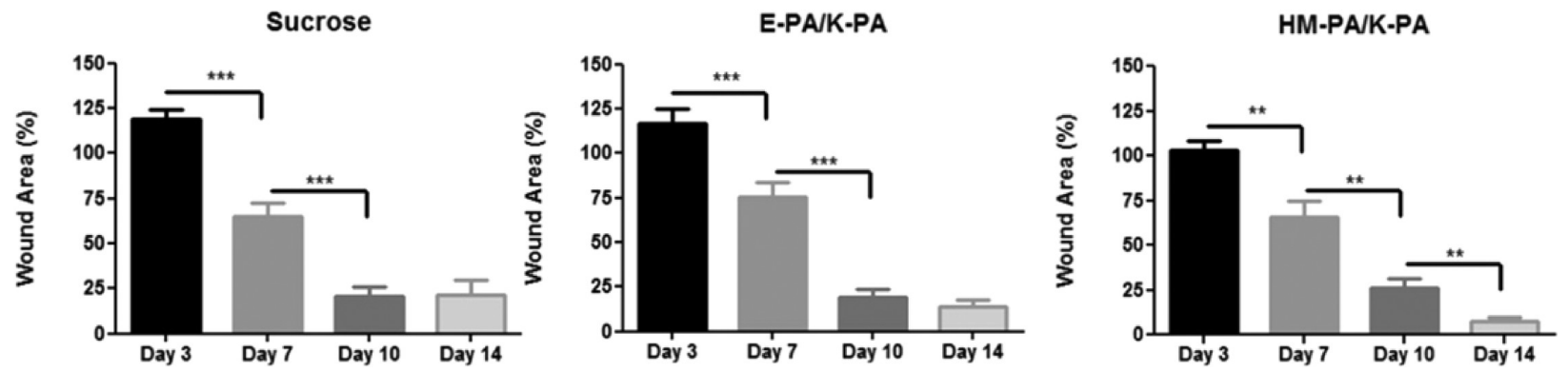

Figure 3. Wound area ratios of (a) sucrose-, (b) E-PA/K-PA-, and (c) HM-PA/K-PA-treated wounds on days 3, 7, 10 , and 14 to that of day $0 . * p<$ 0.05 .

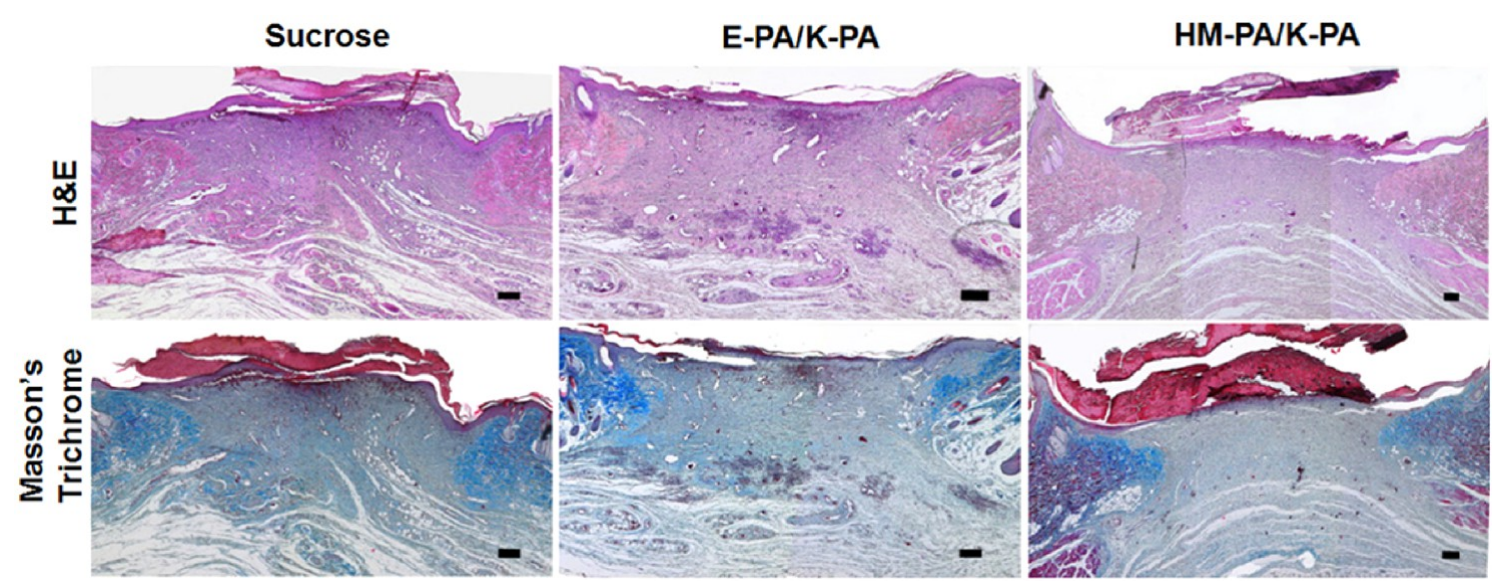

Figure 4. Hematoxylin \& eosin (upper panel) and Masson's trichrome staining (lower panel) of sucrose, E-PA/K-PA, and HM-PA/K-PA treatment on day 7. Scale bars are $200 \mu \mathrm{m}$.

antimouse secondary antibody (1:500; Millipore). Antibody labeling was followed by $3,3^{\prime}$-diaminobenzidine (DAB) staining and hematoxylin counterstaining. Mounting was performed for all samples using a xylene-based mounting medium. Digital images were acquired using a Zeiss Axio Scope Al microscope. Granulation tissue areas of the samples were measured with ImageJ, blood vessel numbers were quantified from sections stained with anti-von Willebrand factor antibody. Re-epithelization was quantified by measuring the distance between left and right wound edges; total re-epithelized tissue area was taken as the sum of new epithelium produced at the left and right edges of the wound. Images were acquired using $10 x$ or $20 x$ objectives and analyzed with ImageJ.

Statistical Analysis. Statistical analyses were performed by using GraphPad Prism 5. One-way ANOVA with Bonferroni multiple comparisons test was employed to determine statistical differences between groups. The level of significance was set at $p<0.05$. Error bars indicate standard error of mean.

\section{RESULTS}

The HM-PA nanofibers (bioactive peptide, lauryl$\operatorname{VVAGEGDK}(\mathrm{pbs}) \mathrm{S}-\mathrm{Am})$ mimic the activity of heparan sulfates by presenting sulfonate, hydroxyl and carboxylate groups on amino acid side chains (Figure 1). The HM-PA and E-PA (negatively charged nonbioactive peptide, Lauryl-VVAGE) molecules form gels through charge neutralization when mixed with K-PA (positively charged nonbioactive peptide, Lauryl-VVAGK-Am) molecule at physiological $\mathrm{pH}$. All peptide amphiphile molecules were characterized by liquid chromatography-mass spectrometry (LC-MS) and purified by preparative HPLC (Figures S1-S3). SEM images of HM-PA/K-PA and E-PA/K-PA networks exhibited porous and nanofibrous structures that resemble the natural architecture of the ECM
(Figure 2a, b). Mechanical properties of the peptide amphiphile gels were analyzed by oscillatory rheology measurements and both HM-PA/K-PA and E-PA/K-PA scaffolds were found to have higher storage moduli $\left(G^{\prime}\right)$ than loss moduli $\left(G^{\prime \prime}\right)$, suggesting that both materials are gels (Figure 2c).

The HM-PA/K-PA and E-PA/K-PA gels were applied topically on full-thickness excision wounds immediately and $24 \mathrm{~h}$ after injury. Wounds were observed to be normal and no evidence of infection was observed during the experimental period. Treatment groups were distributed evenly across distal, median and proximal wounds to eliminate the potential effect of wound site on the healing process. Wound areas were measured on day $3,7,10$, and 14 (Figure S4) to evaluate the recovery associated with each treatment and wound location. The HM-PA/K-PA treated wounds showed sustained healing process even after day $10(p<0.01)$ and reached to $93 \%$ wound closure on day 14, while the recovery of sucrose and E$\mathrm{PA} / \mathrm{K}-\mathrm{PA}$ treated wounds stopped on day 14 and were 79 and $86 \%$, respectively (Figure 3 ). Wound healing rates of different wound locations were also compared to observe the effect of wound placement on healing, but no significant differences were observed among the experimental groups, except between E-PA/K-PA and HM-PA/K-PA on day 3 in the proximal wound area (Figure S5).

In normal wound healing, the proliferation phase peaks at 7 days following the injury and is characterized by granulation tissue formation, re-epithelization and angiogenesis. H\&E and Masson's trichrome stainings of wound areas revealed that the HM-PA/K-PA gel-treated group exhibits a collagen-rich granulation tissue that is less edematous and better organized compared to controls on day 7 (Figure 4). Although 


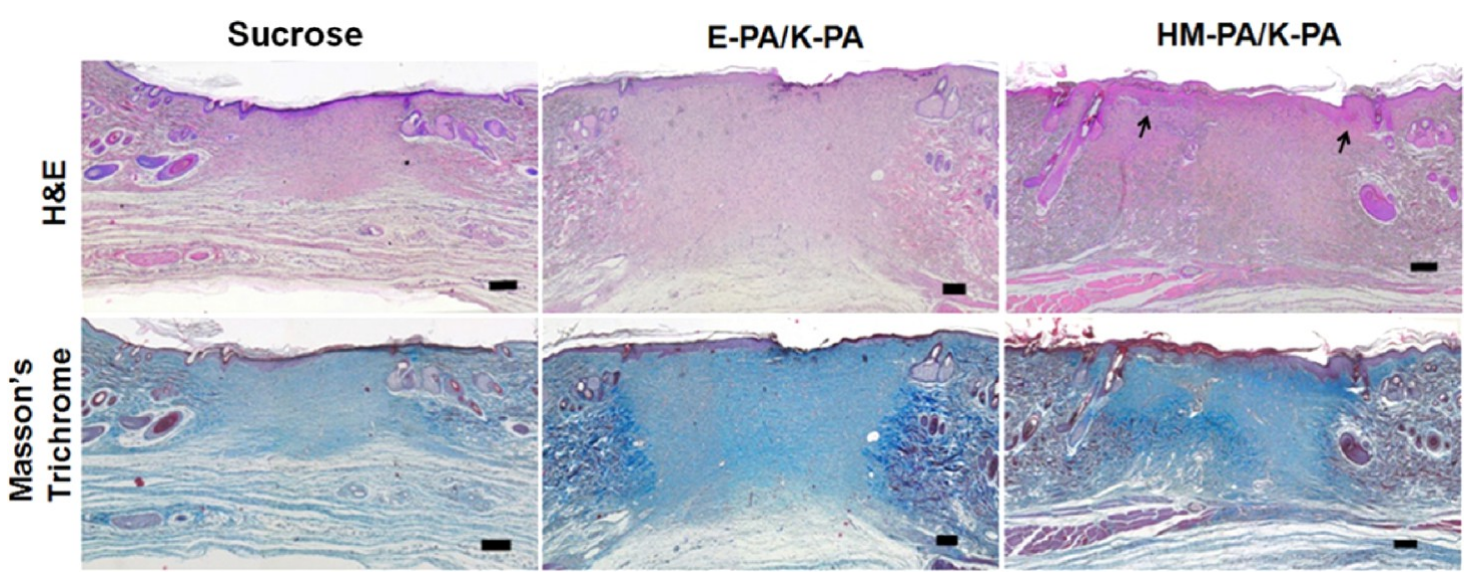

Figure 5. Hematoxylin \& eosin (upper panel) and Masson's Trichrome staining (lower panel) of sucrose, E-PA/K-PA, and HM-PA/K-PA application on day 14. Black arrows indicate rete ridge formation. Scale bars are $200 \mu \mathrm{m}$.
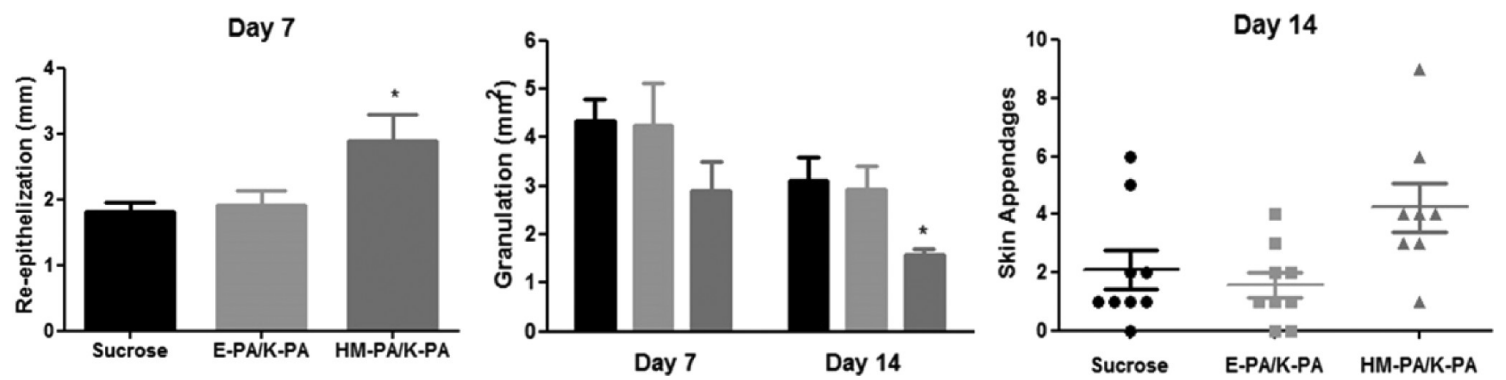

Figure 6. Quantitative analysis of (a) re-epithelization, (b) granulation tissue, and (c) skin appendage number of wound tissues.

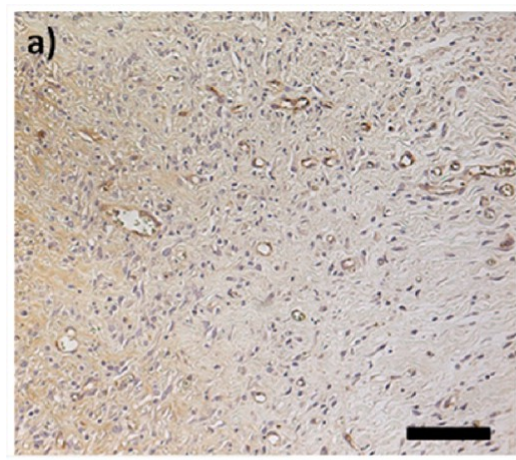

d)

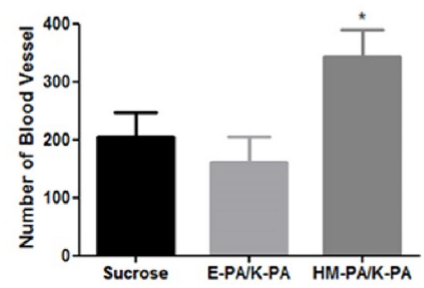

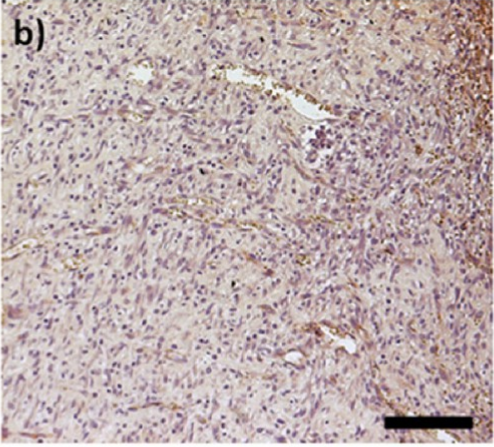

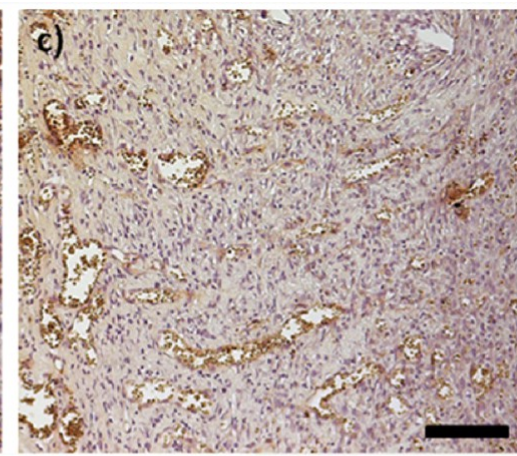

e)

Day 14

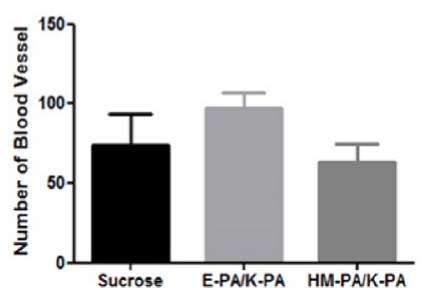

Figure 7. $(\mathrm{a}-\mathrm{c})$ Staining of blood vessels by anti-von Willebrand factor and (d, e) quantification of blood vessels on days 7 and 14 . Representative sections obtained from (a) sucrose, (b) E-PA/K-PA, and (c) HM-PA/K-PA on day 7, 200× magnification. Scale bars are $100 \mu \mathrm{m}$.

granulation areas of all samples were comparable in size (Figure $6 \mathrm{~b})$, the granulation tissue of E-PA/K-PA and control groups had less uniform connective tissue and poorly organized collagen fibers on day 7 (Figure 4). Although a homogeneous, thick, and well-organized granulation tissue could be observed in control groups by day 14 (Figure 5), the HM-PA/K-PAtreated wounds exhibited decreased granulation tissue area and developed a relatively thick basket-weave network of collagen fibers.

Granulation tissue also stimulates uninjured keratinocytes to migrate through the wound area to form a new epithelial layer; as such, re-epithelialization was also quantified by measuring the epithelium advancing from wound edges. HM-PA/K-PA treated wounds displayed more advanced re-epithelialization on day 7 compared to E-PA/K-PA and sucrose groups (Figure 6a). 
Wounds were fully re-epithelialized on day 14 for all experimental groups. Skin appendages were observed to form in all of the HM-PA/K-PA gel treated wounds, whereas control animals lacked skin appendage formation.

Because HM-PA was previously shown to enhance angiogenesis both in vitro and in vivo, and angiogenesis is vital for scar-free wound healing process, we analyzed angiogenesis in tissue sections in order to analyze whether the enhancement in gross morphology of the HM-PA treated groups was due to increased angiogenesis. Anti-von Willebrand factor staining and vascularization assessments were also performed to study the angiogenic responses (Figure 7). Importantly, these results showed that HM-PA/K-PA treatment promoted neovascularization more than both nonbioactive nanofiber treatment and sucrose treatment on day 7 (Figure 7). This increase in blood vessel number was normalized to control treatment groups on day 14, which suggests that the enhanced angiogenesis was not pathological.

\section{DISCUSSION}

Effective wound healing is a major concern for modern medicine, and even fully closed wounds do not fully regain their former functionality due to scar formation. ${ }^{35}$ Although skin substitutes are commonly used to reduce scarring, their utility is limited by issues such as reduced vascularization, mechanical instability, handling issues, lack of biocompatibility, and costs. Artificial scaffolds, which restore the structural and functional properties of skin by modulating the wound repair process, are attractive alternatives to traditional skin substitutes. ${ }^{40}$ In particular, hydrogel-based scaffolds are promising materials for this purpose. ${ }^{4-43}$

In this study, we utilized a bioactive peptide nanofiber system to promote the wound healing process while minimizing scar formation. These nanofibers are designed to mimic heparin and were previously shown to enhance angiogenesis in vitro and in vivo by increasing the local concentration of angiogenic growth factors. ${ }^{38,39}$ The peptide amphiphile molecules are composed of a hydrophobic alkyl tail conjugated to hydrophilic amino acid units and can form self-assembled structures through noncovalent interactions (Figure 1). ${ }^{44,45}$ In our previous studies, it has been shown that nanofibers of ca. $20-30 \mathrm{~nm}$ diameters were formed upon mixing positively charged K-PA molecules and negatively charged HM-PA or E-PA molecules, ${ }^{38,46}$ which self-assemble through electrostatic interactions, $\beta$-sheet formation, and hydrophobic collapse. The $\beta$-sheet-driven nanofiber elongation was provided by the VVAG motif, as the valine amino acid has a high tendency to form $\beta$-sheets. ${ }^{45}$ The wound healing process requires the isolation of the wound from the outside environment in addition to high porosity for gas exchange, and peptide nanofibers are able to satisfy both criteria by forming biodegradable porous gel networks. In addition, the bioactive epitopes presented on nanofiber surfaces further allowed the modulation of cell behavior at the wound site (Figure 2).

Wounded area treated with the HM-PA gels continued to decrease after day 10, whereas wound closure stopped in control groups on day 10 (Figure S4). The main challenge of wound healing is enhancing regeneration while minimizing scar formation, and thus the continuation of wound repair after day 10 may suggest that the HM-PA gel was able to facilitate the scar-free healing of skin wounds.

Skin scars are generally characterized by the deposition of fibrotic tissue, reduced epidermal appendages, alterations in collagen organization and a smooth appearance. ${ }^{10,47}$ Consequently, we investigated the histological appearance of regenerating skin tissue on days 7 and 14 (which mark the points at which proliferation peaks and remodeling starts, respectively) to observe scar formation in the presence and absence of bioactive PA nanofibers. Histological analyses showed that HM-PA/K-PA-treated wounds exhibited a decrease in granulation tissue on day 14 and closely resembled the original tissue in structure (Figures 5 and $6 \mathrm{~b}$ ). Reepithelization also increased on day 7 following HM-PA/K-PA treatment (Figure 6a) and was completed by day 14 .

Interestingly, there was a trend for enhanced skin appendage regeneration in the HM-PA/K-PA gel treated wounds compared control groups. Skin appendages can be regenerated in partial thickness wounds, but not in full thickness wounds. ${ }^{48}$ Although Wnt signaling triggers de novo hair follicle production from epidermal progenitor cells, ${ }^{49}$ skin appendages are nonetheless reduced in wounded tissues. ${ }^{10,11}$ This downward tendency may result in lower densities of rete ridge in wounded tissues. In our study, HM-PA/K-PA treatment was observed to increase rete ridges (Figure 5, black arrows). ${ }^{50}$ Reduced or diminished angiogenesis is one of the main issues in therapeutic approaches for wound healing, especially for maintaining the long-term survival of skin grafts, as the rapid vascularization of the wound area is difficult to achieve. It has been shown that annexin A5 expression, which is a late apoptotic marker, leads the cell death in reduced angiogenesis which is found in impaired wounds. ${ }^{51}$ Several growth factors are known to modulate angiogenesis during wound healing. Damaged endothelial cells secrete fibroblast growth factor 2 $(\text { FGF-2 })^{6,52}$ and inhibiting FGF-2 activity in wounded areas prevents angiogenesis. In addition, it is known that FGF-2 is involved in scarless healing ${ }^{47}$ and affects the vascularization of artificial derma. ${ }^{52,53}$ The other key regulator of angiogenesis is vascular endothelial growth factor (VEGF), which has been reported to promote wound healing in diabetic animal models. $^{52,54}$ In addition to FGF-2 and VEGF, hepatocyte growth factor (HGF) is important for neovascularization during wound healing, and assists in the repair of the epithelial layer by regulating cell growth and motility. ${ }^{55-57}$ The HM-PA nanofibers have the ability to induce capillary-like structures in vitro and in vivo through its strong binding affinity to HGF, VEGF, and FGF-2. ${ }^{38,39}$ To investigate whether the improvements in skin regeneration is promoted by the increased formation of new blood vessels within the granulation area, we investigated angiogenesis in injured tissues on day 7 (Figure 6). There was a significant increase in the number of blood vessels in HM-PA/ K-PA-treated wounds on day 7 compared to nonbioactive nanofiber and no treatment control, which suggests that the increased healing capacity of the HM-PA/K-PA-treated wounds might be due to increased angiogenesis. Furthermore, the regression of angiogenesis, which is required for the latter phases of wound healing, was observed in both HM-PA and control groups by day 14. Fetal wounds heal without scarring and some studies have suggested that decreased angiogenesis can cause scarless healing, ${ }^{5-60}$ and thus, timely regression of the capillary network is advantageous for the peptide nanofiber gel enhanced wound healing process. The PA combinations used in this study were mixed and incubated prior to characterization or application in order to provide homogeneous and mature gel formation. However, because PA gels are injectable, using a double syringe method may provide ease of implementation for future applications. 


\section{CONCLUSION}

In this study, we showed that heparin-mimetic peptide amphiphile nanofiber gels are useful for inproving the functional wound healing process using a rat full thickness wound model. The HM-PA nanofiber gels exhibit features similar to native ECM. Although wound closure rates of the wounds treated with HM-PA/K-PA, E-PA/K-PA, and sucrose combinations were similar, the HM-PA/K-PA group exhibited faster regeneration and better organization of the wound area as indicated by an increasing trend of skin appendages and rete ridge formations. In addition, significant differences in reepithelization and granulation tissue formation rates were observed between bioactive and control groups on day 14 and day 7 , respectively. Furthermore, increased density of newly formed blood vessels in the bioactive PA-treated group on day 7 , which was followed by regression by day 14 , suggests that a rapid course of regeneration is followed in HM-PA-treated groups. Because prompt wound healing is crucial for protecting the wounded area, heparin-mimetic peptide nanofibers may be utilized as a novel therapeutic approach for regenerative wound healing with minimal scar formation.

\section{ASSOCIATED CONTENT}

\section{(S Supporting Information}

The Supporting Information is available free of charge on the ACS Publications website at DOI: 10.1021/acsbiomaterials.6b00165.

LC-MS characterizations, representative images of wound closure, wound area ratios (PDF)

\section{AUTHOR INFORMATION}

\section{Corresponding Authors}

*E-mail: moguler@unam.bilkent.edu.tr.

*E-mail: atekinay@bilkent.edu.tr.

\section{Notes}

The authors declare no competing financial interest.

\section{ACKNOWLEDGMENTS}

We thank Z. Erdogan and I. Ulusoy for helping with HPLC and LC-MS and animal experiments, respectively. This study was supported by the Scientific and Technological Research Council of Turkey (TUBITAK) Grant Number 213M682. M.O.G and A.B.T. acknowledge support from the Turkish Academy of Sciences Distinguished Young Scientist Award (TUBA-GEBIP).

\section{REFERENCES}

(1) Cross, S. E.; Roberts, M. S. Defining a model to predict the distribution of topically applied growth factors and other solutes in excisional full-thickness wounds. J. Invest. Dermatol. 1999, 112 (1), $36-41$.

(2) Yildirimer, L.; Thanh, N. T.; Seifalian, A. M. Skin regeneration scaffolds: a multimodal bottom-up approach. Trends Biotechnol. 2012, 30 (12), 638-648.

(3) Bayat, A.; McGrouther, D. A.; Ferguson, M. W. Skin scarring. BMJ. 2003, 326 (7380), 88-92.

(4) Schreml, S.; Szeimies, R. M.; Karrer, S.; Heinlin, J.; Landthaler, M.; Babilas, P. The impact of the $\mathrm{pH}$ value on skin integrity and cutaneous wound healing. J. Eur. Acad. Dermatol. Venereol. 2010, 24 (4), 373-378.

(5) Singer, A. J.; Clark, R. A. Cutaneous wound healing. N. Engl. J. Med. 1999, 341 (10), 738-746.
(6) Clark, R. The Molecular and Cellular Biology of Wound Repair; Springer Science \& Business Media: New York, 2013.

(7) Adolphe, C.; Wainwright, B. Pathways to improving skin regeneration. Expert Rev. Mol. Med. 2005, 7 (20), 1-14.

(8) Gurtner, G. C.; Werner, S.; Barrandon, Y.; Longaker, M. T. Wound repair and regeneration. Nature 2008, 453 (7193), 314-321.

(9) Markeson, D.; Pleat, J. M.; Sharpe, J. R.; Harris, A. L.; Seifalian, A. M.; Watt, S. M. Scarring, stem cells, scaffolds and skin repair. J. Tissue Eng. Regener. Med. 2015, 9 (6), 649-68.

(10) Martin, P. Wound healing-aiming for perfect skin regeneration. Science 1997, 276 (5309), 75-81.

(11) Miller, M. C.; Nanchahal, J. Advances in the modulation of cutaneous wound healing and scarring. BioDrugs 2005, 19 (6), 363381.

(12) Invernici, G.; Emanueli, C.; Madeddu, P.; Cristini, S.; Gadau, S.; Benetti, A.; Ciusani, E.; Stassi, G.; Siragusa, M.; Nicosia, R.; Peschle, C.; Fascio, U.; Colombo, A.; Rizzuti, T.; Parati, E.; Alessandri, G. Human fetal aorta contains vascular progenitor cells capable of inducing vasculogenesis, angiogenesis, and myogenesis in vitro and in a murine model of peripheral ischemia. Am. J. Pathol. 2007, 170 (6), $1879-1892$.

(13) Delgado, L. M.; Bayon, Y.; Pandit, A.; Zeugolis, D. I. To crosslink or not to cross-link? Cross-linking associated foreign body response of collagen-based devices. Tissue Eng., Part B 2015, 21 (3), $298-313$.

(14) Oberringer, M.; Meins, C.; Bubel, M.; Pohlemann, T. In vitro wounding: effects of hypoxia and transforming growth factor $\beta 1$ on proliferation, migration and myofibroblastic differentiation in an endothelial cell-fibroblast co-culture model. J. Mol. Histol. 2008, 39 (1), 37-47.

(15) Freudenberg, U.; Zieris, A.; Chwalek, K.; Tsurkan, M. V.; Maitz, M. F.; Atallah, P.; Levental, K. R.; Eming, S. A.; Werner, C. Heparin desulfation modulates VEGF release and angiogenesis in diabetic wounds. J. Controlled Release 2015, 220, 79-88.

(16) Eming, S. A.; Martin, P.; Tomic-Canic, M. Wound repair and regeneration: mechanisms, signaling, and translation. Sci. Transl. Med. 2014, 6 (265), $265 \mathrm{sr} 6$.

(17) Dvir, T.; Timko, B. P.; Kohane, D. S.; Langer, R. Nanotechnological strategies for engineering complex tissues. Nat. Nanotechnol. 2011, 6 (1), 13-22.

(18) Fairbrother, W. J.; Champe, M. A.; Christinger, H. W.; Keyt, B. A.; Starovasnik, M. A. Solution structure of the heparin-binding domain of vascular endothelial growth factor. Structure 1998, 6 (5), 637-648.

(19) Hynes, R. O. The extracellular matrix: not just pretty fibrils. Science 2009, 326 (5957), 1216-1219.

(20) Koolwijk, P.; van Erck, M. G.; de Vree, W. J.; Vermeer, M. A.; Weich, H. A.; Hanemaaijer, R; van Hinsbergh, V. W. Cooperative effect of TNFalpha, bFGF, and VEGF on the formation of tubular structures of human microvascular endothelial cells in a fibrin matrix. Role of urokinase activity. J. Cell Biol. 1996, 132 (6), 1177-1188.

(21) Pellegrini, L. Role of heparan sulfate in fibroblast growth factor signalling: a structural view. Curr. Opin. Struct. Biol. 2001, 11 (5), 629634.

(22) Schlessinger, J.; Plotnikov, A. N.; Ibrahimi, O. A.; Eliseenkova, A. V.; Yeh, B. K.; Yayon, A.; Linhardt, R. J.; Mohammadi, M. Crystal structure of a ternary FGF-FGFR-heparin complex reveals a dual role for heparin in FGFR binding and dimerization. Mol. Cell 2000, 6 (3), $743-750$.

(23) Hoeben, A.; Landuyt, B.; Highley, M. S.; Wildiers, H.; Van Oosterom, A. T.; De Bruijn, E. A. Vascular endothelial growth factor and angiogenesis. Pharmacol Rev. 2004, 56 (4), 549-580.

(24) Hartgerink, J. D.; Beniash, E.; Stupp, S. I. Self-assembly and mineralization of peptide-amphiphile nanofibers. Science 2001, 294 (5547), 1684-1688.

(25) Stendahl, J. C.; Rao, M. S.; Guler, M. O.; Stupp, S. I. Intermolecular Forces in the Self-Assembly of Peptide Amphiphile Nanofibers. Adv. Funct. Mater. 2006, 16 (4), 499-508. 
(26) Jiang, H.; Guler, M. O.; Stupp, S. I. The internal structure of self-assembled peptide amphiphiles nanofibers. Soft Matter 2007, 3 (4), 454-462.

(27) Cui, H.; Webber, M. J.; Stupp, S. I. Self-assembly of peptide amphiphiles: from molecules to nanostructures to biomaterials. Biopolymers 2010, 94 (1), 1-18.

(28) Mammadov, B.; Mammadov, R.; Guler, M. O.; Tekinay, A. B. Cooperative effect of heparan sulfate and laminin mimetic peptide nanofibers on the promotion of neurite outgrowth. Acta Biomater. 2012, 8 (6), 2077-2086.

(29) Silva, G. A.; Czeisler, C.; Niece, K. L.; Beniash, E.; Harrington, D. A.; Kessler, J. A.; Stupp, S. I. Selective differentiation of neural progenitor cells by high-epitope density nanofibers. Science 2004, 303 (5662), 1352-1355.

(30) Berns, E. J.; Alvarez, Z.; Goldberger, J. E.; Boekhoven, J.; Kessler, J. A.; Kuhn, H. G.; Stupp, S. I. A tenascin-C mimetic peptide amphiphile nanofiber gel promotes neurite outgrowth and cell migration of neurosphere-derived cells. Acta Biomater. 2016, 37, 50.

(31) Murphy, M. B.; Blashki, D.; Buchanan, R. M.; Fan, D.; De Rosa, E.; Shah, R. N.; Stupp, S. I.; Weiner, B. K.; Simmons, P. J.; Ferrari, M.; Tasciotti, E. Multi-composite bioactive osteogenic sponges featuring mesenchymal stem cells, platelet-rich plasma, nanoporous silicon enclosures, and Peptide amphiphiles for rapid bone regeneration. J. Funct. Biomater. 2011, 2 (2), 39-66.

(32) Uzunalli, G.; Soran, Z.; Erkal, T. S.; Dagdas, Y. S.; Dinc, E.; Hondur, A. M.; Bilgihan, K.; Aydin, B.; Guler, M. O.; Tekinay, A. B. Bioactive self-assembled peptide nanofibers for corneal stroma regeneration. Acta Biomater. 2014, 10 (3), 1156-1166.

(33) Huang, Z.; Newcomb, C. J.; Lei, Y.; Zhou, Y.; Bornstein, P.; Amendt, B. A.; Stupp, S. I.; Snead, M. L. Bioactive nanofibers enable the identification of thrombospondin 2 as a key player in enamel regeneration. Biomaterials 2015, 61, 216-28.

(34) Shah, R. N.; Shah, N. A.; Del Rosario Lim, M. M.; Hsieh, C.; Nuber, G.; Stupp, S. I. Supramolecular design of self-assembling nanofibers for cartilage regeneration. Proc. Natl. Acad. Sci. U. S. A. 2010, 107 (8), 3293-3298.

(35) Zha, R. H.; Sur, S.; Boekhoven, J.; Shi, H. Y.; Zhang, M.; Stupp, S. I. Supramolecular assembly of multifunctional maspin-mimetic nanostructures as a potent peptide-based angiogenesis inhibitor. Acta Biomater. 2015, 12, 1-10.

(36) Chow, L. W.; Bitton, R.; Webber, M. J.; Carvajal, D.; Shull, K. R.; Sharma, A. K.; Stupp, S. I. A bioactive self-assembled membrane to promote angiogenesis. Biomaterials 2011, 32 (6), 1574-1582.

(37) Rajangam, K.; Behanna, H. A.; Hui, M. J.; Han, X.; Hulvat, J. F.; Lomasney, J. W.; Stupp, S. I. Heparin binding nanostructures to promote growth of blood vessels. Nano Lett. 2006, 6 (9), 2086-90.

(38) Mammadov, R.; Mammadov, B.; Toksoz, S.; Aydin, B.; Yagci, R.; Tekinay, A. B.; Guler, M. O. Heparin mimetic peptide nanofibers promote angiogenesis. Biomacromolecules 2011, 12 (10), 3508-19.

(39) Mammadov, R.; Mammadov, B.; Guler, M. O.; Tekinay, A. B. Growth factor binding on heparin mimetic peptide nanofibers. Biomacromolecules 2012, 13 (10), 3311-9.

(40) Metcalfe, A. D.; Ferguson, M. W. Tissue engineering of replacement skin: the crossroads of biomaterials, wound healing, embryonic development, stem cells and regeneration. J. R. Soc., Interface 2007, 4 (14), 413-437.

(41) Ghosh, K.; Ren, X. D.; Shu, X. Z.; Prestwich, G. D.; Clark, R. A. Fibronectin functional domains coupled to hyaluronan stimulate adult human dermal fibroblast responses critical for wound healing. Tissue Eng. 2006, 12 (3), 601-13.

(42) Helary, C.; Bataille, I.; Abed, A.; Illoul, C.; Anglo, A.; Louedec, L.; Letourneur, D.; Meddahi-Pelle, A.; Giraud-Guille, M. M. Concentrated collagen hydrogels as dermal substitutes. Biomaterials 2010, 31 (3), 481-490.

(43) Zhong, S.; Teo, W. E.; Zhu, X.; Beuerman, R.; Ramakrishna, S.; Yung, L. Y. Formation of collagen-glycosaminoglycan blended nanofibrous scaffolds and their biological properties. Biomacromolecules 2005, 6 (6), 2998-3004.
(44) Hartgerink, J. D.; Beniash, E.; Stupp, S. I. Peptide-amphiphile nanofibers: a versatile scaffold for the preparation of self-assembling materials. Proc. Natl. Acad. Sci. U. S. A. 2002, 99 (8), 5133-5138.

(45) Niece, K. L.; Hartgerink, J. D.; Donners, J. J.; Stupp, S. I. Selfassembly combining two bioactive peptide-amphiphile molecules into nanofibers by electrostatic attraction. J. Am. Chem. Soc. 2003, 125 (24), $7146-7$.

(46) Uzunalli, G.; Tumtas, Y.; Delibasi, T.; Yasa, O.; Mercan, S.; Guler, M. O.; Tekinay, A. B. Improving pancreatic islet in vitro functionality and transplantation efficiency by using heparin mimetic peptide nanofiber gels. Acta Biomater. 2015, 22, 8-18.

(47) Paramonov, S. E.; Jun, H. W.; Hartgerink, J. D. Self-assembly of peptide-amphiphile nanofibers: the roles of hydrogen bonding and amphiphilic packing. J. Am. Chem. Soc. 2006, 128 (22), 7291-8.

(48) Kweon, D. K.; Song, S. B.; Park, Y. Y. Preparation of watersoluble chitosan/heparin complex and its application as wound healing accelerator. Biomaterials 2003, 24 (9), 1595-1601.

(49) Ito, M.; Yang, Z.; Andl, T.; Cui, C.; Kim, N.; Millar, S. E.; Cotsarelis, G. Wnt-dependent de novo hair follicle regeneration in adult mouse skin after wounding. Nature 2007, 447 (7142), 316-320.

(50) Mannor, G. E.; Phelps, R. G.; Friedman, A. H.; Meltzer, M. Eyelid healing after carbon dioxide laser skin resurfacing: histological analysis. Arch. Ophthalmol. 1999, 117 (7), 913-916.

(51) Krisp, C.; Jacobsen, F.; McKay, M. J.; Molloy, M. P.; Steinstraesser, L.; Wolters, D. A. Proteome analysis reveals antiangiogenic environments in chronic wounds of diabetes mellitus type 2 patients. Proteomics 2013, 13 (17), 2670-81.

(52) Das, S.; Singh, G.; Monteforte, A.; Martinez, M.; Wright, C.; Martin, P.; Dunn, A.; Baker, A. Syndesome-Based Dressings for Enhanced Wound Healing in Diabetic Ulcers. Arterioscler., Thromb., Vasc. Biol. 2015, 35, A54.

(53) Kawai, K.; Suzuki, S.; Tabata, Y.; Ikada, Y.; Nishimura, Y. Accelerated tissue regeneration through incorporation of basic fibroblast growth factor-impregnated gelatin microspheres into artificial dermis. Biomaterials 2000, 21 (5), 489-499.

(54) Galiano, R. D.; Tepper, O. M.; Pelo, C. R.; Bhatt, K. A.; Callaghan, M.; Bastidas, N.; Bunting, S.; Steinmetz, H. G.; Gurtner, G. C. Topical vascular endothelial growth factor accelerates diabetic wound healing through increased angiogenesis and by mobilizing and recruiting bone marrow-derived cells. Am. J. Pathol. 2004, 164 (6), 1935-1947.

(55) Conway, K.; Price, P.; Harding, K. G.; Jiang, W. G. The molecular and clinical impact of hepatocyte growth factor, its receptor, activators, and inhibitors in wound healing. Wound Repair Regen. 2006, 14 (1), 2-10.

(56) Islam, M. S.; Zhou, H. Isolation and characterization of putative epidermal stem cells derived from Cashmere goat fetus. Eur. J. Dermatol. 2007, 17 (4), 302-308.

(57) Matsumoto, K.; Nakamura, T. Hepatocyte growth factor (HGF) as a tissue organizer for organogenesis and regeneration. Biochem. Biophys. Res. Commun. 1997, 239 (3), 639-644.

(58) Borena, B. M.; Martens, A.; Broeckx, S. Y.; Meyer, E.; Chiers, K.; Duchateau, L.; Spaas, J. H. Regenerative Skin Wound Healing in Mammals: State-of-the-Art on Growth Factor and Stem Cell Based Treatments. Cell. Physiol. Biochem. 2015, 36 (1), 1-23.

(59) Ihara, S.; Motobayashi, Y.; Nagao, E.; Kistler, A. Ontogenetic transition of wound healing pattern in rat skin occurring at the fetal stage. Development 1990, 110 (3), 671-680.

(60) Whitby, D. J.; Ferguson, M. W. Immunohistochemical localization of growth factors in fetal wound healing. Dev. Biol. 1991, 147 (1), 207-215. 